\title{
Serum angiotensin-converting enzyme 2 is an independent risk factor for in-hospital mortality following open surgical repair of ruptured abdominal aortic aneurysm
}

\author{
WANPIN NIE*, YAN WANG ${ }^{*}$, KAI YAO, ZHENG WANG and HAO WU \\ Department of Vascular Surgery, The Third Xiangya Hospital, Central South University, Changsha, Hunan 410013, P.R. China
}

Received June 18, 2015; Accepted April 21, 2016

DOI: $10.3892 /$ etm. 2016.3466

\begin{abstract}
Open surgical repair (OSR) is a conventional surgical method used in the repair a ruptured abdominal aortic aneurysm (AAA); however, OSR results in high perioperative mortality rates. The level of serum angiotensin-converting enzyme 2 (ACE2) has been reported to be an independent risk factor for postoperative in-hospital mortality following major cardiopulmonary surgery. In the present study, the association of serum ACE2 levels with postoperative in-hospital mortality was investigated in patients undergoing OSR for ruptured AAA. The study enrolled 84 consecutive patients underwent OSR for ruptured AAA and were subsequently treated in the intensive care unit. Patients who succumbed postoperatively during hospitalization were defined as non-survivors. Serum ACE2 levels were measured in all patients prior to and following the surgery using ELISA kits. The results indicated that non-survivors showed significantly lower mean preoperative and postoperative serum ACE2 levels when compared with those in survivors. Multivariate logistic regression analysis also showed that, subsequent to adjusting for potential confounders, the serum ACE2 level on preoperative day 1 showed a significant negative association with the postoperative in-hospital mortality. This was confirmed by multivariate hazard ratio analysis, which showed that, subsequent to adjusting for the various potential confounders, the risk of postoperative in-hospital mortality remained significantly higher in the two lowest serum ACE2 level quartiles compared with that in the highest quartile on preoperative day 1 . In conclusion, the present study provided the first evidence supporting that the serum ACE2 level is an independent risk factor for the in-hospital
\end{abstract}

Correspondence to: Dr Kai Yao, Department of Vascular Surgery, The Third Xiangya Hospital, Central South University, 138 Tongzipo Road, Changsha, Hunan 410013, P.R. China

E-mail: xiangyayaokai2014@gmail.com

${ }^{*}$ Contributed equally

Key words: angiotensin-converting enzyme 2, open surgical repair, abdominal aortic aneurysm, rupture, in-hospital mortality, hazard ratio, odds ratio mortality following OSR for ruptured AAA. Furthermore, low serum ACE2 levels on preoperative day 1 were found to be associated with increased postoperative in-hospital mortality. Therefore, the serum ACE2 level on preoperative day 1 may be a potential biomarker or prognostic factor for in-hospital mortality following OSR for ruptured AAA.

\section{Introduction}

Abdominal aortic aneurysm (AAA) involves the dilation of the abdominal aorta and occurs in up to $9 \%$ of adults with an age of $>65$ years in Western countries (1). The mortality resulting from ruptured AAA remains $\leq 90 \%$ despite medical advances (2). Currently, the only treatment for AAA is surgery, and approximately $48 \%$ of the perioperative mortality rate is associated with surgical repair of ruptured AAA (3). The conventional method used to repair a ruptured AAA is open surgical repair (OSR) with replacement of the ruptured aneurysm using a synthetic tube graft, which is an extensive repair method that results in a high postoperative mortality rate (4). Patients undergoing OSR for ruptured AAA may succumb to the disease in the intensive care unit (ICU) within days after surgery (5). Thus, the identification of preoperative or early postoperative predictive biomarkers of mortality in these patients may reduce postoperative in-hospital mortality.

The renin-angiotensin system (RAS) serves a crucial role in cardiovascular regulation (6). Angiotensin-converting enzyme 2 (ACE2), which is expressed in the majority of tissues, is known to serve a pivotal role in the body as an endogenous regulator of the RAS (7). Recent studies have demonstrated the value of the serum ACE2 level in the prediction of in-hospital mortality and morbidities subsequent to major cardiopulmonary surgeries $(8,9)$. Wang et al reported that the serum ACE2 level was an independent risk factor for postoperative myocardial infarction and in-hospital mortality following coronary artery bypass grafting (CABG) (8). Furthermore, Li et al reported that the serum ACE2 level was an independent risk factor for in-hospital mortality and major morbidities following major pulmonary resection in non-small cell lung cancer (NSCLC) patients (9). In the present study, the association of serum ACE2 levels with postoperative in-hospital mortality was examined in patients undergoing OSR for ruptured AAA. 
Table I. Baseline general characteristics of patients.

\begin{tabular}{|c|c|c|c|}
\hline Subject Characteristics & Survivors $(\mathrm{n}=51)$ & Non-survivors $(n=33)$ & P-value \\
\hline Age (years) & $69.4 \pm 10.7$ & $73.9 \pm 8.3$ & 0.07 \\
\hline Gender & & & 0.21 \\
\hline Male & $40(78.4)$ & $21(63.6)$ & \\
\hline Female & $11(21.6)$ & $12(36.4)$ & \\
\hline Body mass index $\left(\mathrm{kg} / \mathrm{m}^{2}\right)$ & $28.6 \pm 7.3$ & $29.2 \pm 7.5$ & 0.72 \\
\hline Systolic blood pressure (mmHg) & $130.9 \pm 17.5$ & $139.2 \pm 18.4$ & 0.52 \\
\hline Diastolic blood pressure (mmHg) & $85.2 \pm 11.9$ & $94.6 \pm 13.6$ & 0.33 \\
\hline Current smoker & $15(29.4)$ & $16(48.5)$ & 0.11 \\
\hline Hypertension & $33(64.7)$ & $23(69.7)$ & 0.81 \\
\hline Diabetes mellitus & $10(19.6)$ & $12(36.4)$ & 0.13 \\
\hline Hyperlipidemia & $46(90.2)$ & $30(90.9)$ & $<0.99$ \\
\hline Kidney disease & $3(6.0)$ & $3(9.1)$ & 0.68 \\
\hline Cardiovascular comorbidity & $43(84.3)$ & $29(87.9)$ & 0.76 \\
\hline Pulmonary comorbidity & $16(31.4)$ & $12(36.4)$ & 0.64 \\
\hline
\end{tabular}

Values are expressed as the mean \pm standard deviation for continuous variables (comparisons performed with Student's t-test) and as $\mathrm{n}(\%)$ for categorical variables (comparisons performed with $\chi^{2}$ test).

\section{Patients and methods}

Patients. Between May 2011 and December 2014, 84 consecutive patients who underwent OSR for ruptured AAA and were subsequently treated in the ICU at the Department of Vascular Surgery of the Third Xiangya Hospital, Central South University (Changsha, China) were enrolled in the present study. Baseline characteristics were collected up to preoperative day 1. Baseline systolic and diastolic blood pressure were calculated as the mean values of blood pressure measurements taken from preoperative day 7 to preoperative day 1 using the HEM907XL Professional Digital Blood Pressure Monitor (Omron Co., Ltd., Beijing, China). The initial AAA diameter and iliac and renal artery involvement were assessed at admission and reconfirmed on preoperative days 7-9 using multislice computed tomography (CT; Somatom Definition scanner; Siemens, Munich, Germany). Preoperative medication usage were ascertained from a case history evaluation of the 3 years prior to preoperative day 1 . The body mass index (BMI) and smoking status of all patients was collected on preoperative days 5-7. Hyperlidemia was detected by blood tests at admission. Kidney disease and other comorbidities were collected from the case history at admission. The study was approved by the Ethics Committee of the Third Xiangya Hospital (Central South University), and all the participants provided written informed consent prior to commencing the study.

Surgical procedure. The open surgical procedure was performed according to standard vascular techniques (4), using an anterior transperitoneal approach in all patients under general anesthesia. Subsequent to aortic clamping, the lumbar artery branches were minimally dissected and, when necessary, intrasaccular ligation was also performed. Next, the prosthetic graft was attached to the proximal and distal aspects of the aneurysm by suturing. Aortoaortic 'straight tube' grafts and bifurcated prostheses (Endurant II Stent Graft System; Medtronic, Inc., Minneapolis, MN, USA) were used.

Definition of ruptured $A A A$. AAA was defined as a focal dilation of the abdominal aorta that exceeded $50 \%$ of the original artery diameter and/or was $>3 \mathrm{~cm}$ in diameter $(4,10)$. Ruptured AAA was defined as hemorrhage outside the aortic wall and was diagnosed by CT. In hemodynamically unstable patients, imaging could not be performed and thus the diagnosis was confirmed intraoperatively. Patients who succumbed to the disease postoperatively during hospitalization were classified as non-survivors, and the patients who survived postoperatively during hospitalization were termed survivors. The hospitalization period ranged from 1-3 weeks.

Measurement of serum ACE2 levels. Serum samples were collected from all patients preoperatively on days 3,2 and 1, and postoperatively at $1 \mathrm{~h}$ after surgery and on days 1,2 and 3 . Serum ACE2 levels were measured using an ACE2 (human) ELISA kit (cat. no. K4918-100; BioVision, Inc., Milpitas, CA, USA) according to the manufacturer's instructions. The assay range of detection was 0.391-25 ng ACE2/ml.

Statistical analysis. Continuous variables are expressed as the mean \pm standard deviation and comparisons between the means of the two groups were performed using Student's t-test. Categorical variables are expressed as the number $(\%)$ and were analyzed using the $\chi^{2}$ test. The multivariate Cox proportional hazard model was used to calculate the adjusted hazard ratios (HRs) and 95\% confidence intervals (CIs). In addition, multivariate logistic regression analysis was performed in order to assess the odds ratios (ORs) and 95\% CIs. Statistical analysis was performed using SPSS version 19.0 (IBM Corp., Armonk, NY, USA). A two-tailed $\mathrm{P}<0.05$ was considered to indicate statistically significant difference. 
Table II. Baseline disease characteristics of patients.

\begin{tabular}{lccc}
\hline Subject characteristics & Survivors $(\mathrm{n}=51)$ & Non-survivors $(\mathrm{n}=33)$ & P-value \\
\hline AAA initial diameter $(\mathrm{cm})$ & $5.3 \pm 0.8$ & $6.4 \pm 1.2$ & 0.13 \\
Length of AAA $(\mathrm{cm})$ & $7.3 \pm 2.3$ & $8.5 \pm 2.0$ & 0.62 \\
Left iliac involvement & $7(13.7)$ & $8(24.2)$ & 0.25 \\
Right iliac involvement & $8(15.7)$ & $10(30.3)$ & 0.17 \\
Renal artery involvement & $2(3.9)$ & $3(9.1)$ & 0.38 \\
Medication history & & $27(81.8)$ & 0.79 \\
$\beta$-blocker & $40(78.4)$ & $29(87.9)$ & 0.73 \\
Statin & $46(90.2)$ & $21(63.6)$ & $30(90.9)$ \\
ACE inhibitor/ARB & $29(56.9)$ & $10(30.3)$ & 0.65 \\
Daily aspirin & $45(88.2)$ & $14(27.5)$ & 0.99 \\
Anticoagulant & 140.81 \\
\hline
\end{tabular}

Values are expressed as the mean \pm standard deviation for continuous variables (comparisons performed with Student $\mathrm{t}$-test) and as $\mathrm{n}(\%)$ for categorical variables (comparisons performed with $\chi^{2}$ test). AAA, abdominal aortic aneurysm; ACE, angiotensin-converting enzyme; ARB, angiotensin II receptor blocker.
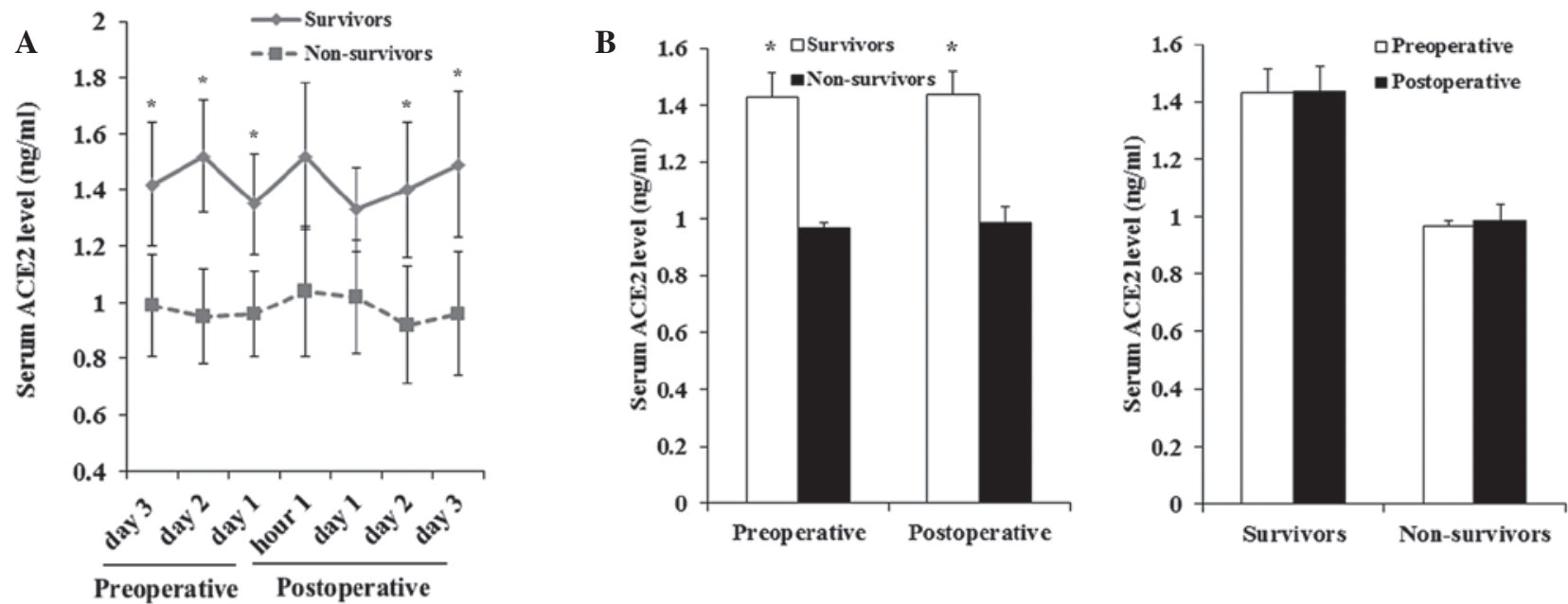

Figure 1. Preoperative and postoperative serum ACE2 levels in survivors and non-survivors. (A) Serum ACE2 levels were measured using a human ACE2 ELISA kit in patients undergoing OSR for ruptured AAA on preoperative days 3,2 and 1, at $1 \mathrm{~h}$ after surgery and at postoperative days 1,2 and 3. The serum ACE2 levels were compared between patients succumbing postoperatively during hospitalization (non-survivors) and patients that survived postoperatively (survivors). (B) Mean preoperative and postoperative serum ACE2 levels were compared between non-survivors and survivors. ${ }^{\mathrm{P}}<0.05$ vs. non-survivors. ACE2, angiotensin-converting enzyme 2.

\section{Results}

Preoperative and postoperative serum ACE2 levels in non-survivors and survivors following $O S R$ for ruptured $A A A$. In the present study, patients succumbing to the disease during the hospitalization period following OSR for ruptured AAA were defined as non-survivors, as opposed to patients that survived. As shown in Table I, there were no statistically significant differences between the non-survivors and survivors regarding parameters including age, gender, BMI, current smoking status and comorbidities $(\mathrm{P}>0.05)$. As shown in Table II, there were no significant differences between the non-survivors and survivors with regard to the baseline disease characteristics, such as the AAA initial diameter, iliac and renal artery involvement, and preoperative medications.
Serum ACE2 levels were measured in all patients on preoperative days 3,2 and 1 , at $1 \mathrm{~h}$ after surgery and on postoperative days 1,2 and 3. A shown in Fig. 1A, the serum ACE2 levels of non-survivors were significantly lower compared with those in survivors, preoperatively and on postoperative days 2 and $3(\mathrm{P}<0.05)$. However, there was no significant difference in the mean serum ACE2 levels prior to and following surgery in non-survivors or in survivors (Fig. 1B). The findings suggested that the serum ACE2 level may be a predictive indicator for the in-hospital mortality following OSR for ruptured AAA, since the level was consistently increased in non-survivors.

Multivariate logistic analysis for risk factors of postoperative in-hospital mortality. Multivariate logistic regression analysis was then performed to identify the risk factors for in-hospital 
Table III. Variables included in multivariate logistic regression analysis.

\begin{tabular}{lll}
\hline Variable & \multicolumn{1}{c}{ Factor } & Value assignment \\
\hline X1 & Age (years) & \\
X2 & Gender & $51-59=1,60-69=2,70-79=3, \geq 80=4$ \\
X3 & Current smoker & Female=1, Male=0 \\
X4 & Diastolic blood pressure $(\mathrm{mmHg})$ & Yes=1, No=0 \\
X5 & Diabetes mellitus & $71-89=1,90-119=2, \geq 120=3$ \\
X6 & Cardiovascular comorbidity & Yes=1, No=0 \\
X7 & Pulmonary comorbidity & Yes=1, No=0 \\
X8 & Kidney disease & Yes=1, No=0 \\
X9 & AAA initial diameter, cm & Yes=1, No=0 \\
X10 & Left iliac involvement & $4.4-4.9=1,5.0-5.9=2,6.0-6.9=3,7.0-7.9=4, \geq 8.0=5$ \\
X11 & Right iliac involvement & Yes=1, No=0 \\
X12 & Renal artery involvement & Yes=1, No=0 \\
X13 & Serum ACE2 at preoperative day $1(\mathrm{ng} / \mathrm{ml})$ & Yes=1, No=0 \\
Y & Postoperative in-hospital survival & $\leq 1.01=1,1.02-1.34=2,1.35-1.79=3, \geq 1.80=4$ \\
\hline
\end{tabular}

AAA, abdominal aortic aneurysm; ACE2, angiotensin-converting enzyme 2.

Table IV. Logistic regression model of risk factors for postoperative in-hospital mortality.

\begin{tabular}{|c|c|c|c|c|c|}
\hline Selected variables & $\begin{array}{c}\text { Point } \\
\text { estimate }\end{array}$ & $\begin{array}{c}\text { Standard } \\
\text { error }\end{array}$ & $\begin{array}{l}\text { Wald } \\
\chi^{2} \text { test }\end{array}$ & P-value & $\begin{array}{l}\text { Odds ratio } \\
(95 \% \mathrm{CI})\end{array}$ \\
\hline Age (years) & 3.52 & 1.35 & 6.40 & 0.011 & $8.67(1.93-29.86)$ \\
\hline Serum ACE2 at preoperative day 1 (ng/ml) & -3.17 & 1.22 & 6.31 & 0.012 & $0.34(0.25-0.82)$ \\
\hline Current smoker & 1.93 & 0.81 & 5.25 & 0.021 & $5.09(1.38-21.45)$ \\
\hline Diastolic blood pressure (mmHg) & 1.54 & 0.73 & 4.11 & 0.045 & $4.29(1.06-18.70)$ \\
\hline
\end{tabular}

Stepwise logistic regression analysis was performed with $\alpha_{\text {in }}=0.05$ and $\alpha_{\text {out }}=0.10$. ACE2, angiotensin-converting enzyme 2; CI, confidence interval.

mortality following OSR for ruptured AAA. The postoperative in-hospital survival status was used as the dependent variable (Y) in the logistic regression analysis (Table III). Cardiovascular comorbidity, pulmonary comorbidity, kidney disease, as well as variables with a $\mathrm{P}<0.50$ in comparisons between non-survivors and survivors (Tables I and II), including age, gender, diastolic blood pressure, current smoking status, diabetes mellitus, AAA initial diameter, left iliac involvement, right iliac involvement and renal artery involvement were selected as independent variables (X1-X13; Table III). Among these, the continuous variables including age, diastolic blood pressure and AAA initial diameter were categorized into groups for more accurate analysis results (Table III) (11). The serum ACE2 level on preoperative day 1 was used as an independent variable (X10), and was divided into the following quartile categories: $\leq 1.01,1.02-1.34$, 1.35-1.79 and $\geq 1.80 \mathrm{ng} / \mathrm{ml}$ (Table III).

As shown in Table IV, the age, current smoking status, serum ACE2 level and diastolic blood pressure were found to be significantly associated with the postoperative in-hospital survival $(\mathrm{P}<0.05)$. Based on the variable value assignment listed in Table III, the results in Table IV indicated that a higher age and diastolic blood pressure was associated with reduced postoperative in-hospital survival. Similarly, a lower serum ACE2 level on preoperative day 1 was associated with lower postoperative in-hospital survival and thus higher postoperative in-hospital mortality. In the entire cohort of patients $(n=84)$, the serum ACE2 levels on preoperative day 1 were not significantly correlated with age $(\mathrm{r}=-0.19 ; \mathrm{P}=0.13)$, or with diastolic $(\mathrm{r}=-0.15 ; \mathrm{P}=0.21)$ and systolic blood pressure $(\mathrm{r}=-0.14 ; \mathrm{P}=0.25)$. Furthermore, there was no statistically significant difference in the serum ACE2 level between current smokers and non-current smokers $(1.2 \pm 0.08$ vs. $1.3 \pm 0.06 ; \mathrm{P}=0.11)$. These results confirmed that the serum ACE2 level may be an independent risk factor for the in-hospital mortality following OSR for ruptured AAA.

Multivariate HR analysis of postoperative in-hospital mortality by serum ACE2 levels. The present study assessed the HRs of postoperative in-hospital mortality according to the serum ACE2 levels. Initially, adjustment was performed for cardiovascular comorbidity, pulmonary comorbidity, kidney disease, age, gender, diastolic blood pressure, current smoking status, diabetes mellitus, AAA initial diameter, left iliac involvement, right iliac involvement and renal artery involvement. As shown 
Table V. Adjusted hazard ratios of postoperative in-hospital mortality according to serum ACE2 levels.

\begin{tabular}{|c|c|c|c|c|c|c|c|}
\hline & \multicolumn{4}{|c|}{ Serum ACE2 levels (ng/ml) } & \multirow{2}{*}{$\begin{array}{c}\text { P-value } \\
\text { (across } \\
\text { categories) }\end{array}$} & \multirow[b]{2}{*}{$\begin{array}{l}\text { Continuous } \\
\text { log scale }\end{array}$} & \multirow[b]{2}{*}{$\begin{array}{c}\text { P-value } \\
\text { (continuous) }\end{array}$} \\
\hline & $\begin{array}{l}\leq 1.01 \\
(n=21)\end{array}$ & $\begin{array}{c}1.02-1.34 \\
(\mathrm{n}=21)\end{array}$ & $\begin{array}{c}1.35-1.79 \\
(n=21)\end{array}$ & $\begin{array}{l}\geq 1.80 \\
(n=21)\end{array}$ & & & \\
\hline Total mortality & $14(66.7)$ & $11(52.4)$ & $6(28.6)$ & $2(9.5)$ & & & \\
\hline $\mathrm{HR}^{\mathrm{b}}(95 \% \mathrm{CI})$ & $6.1(3.3-23.8)$ & $3.4(2.2-12.5)$ & $1.1(0.8-4.1)$ & 1 ( $\left.{ }^{\mathrm{c}} \mathrm{ref}\right)$ & $<0.001$ & $5.5(5.1-6.4)$ & $<0.001$ \\
\hline
\end{tabular}

Table VI. Association of serum ACE2 levels with postoperative in-hospital mortality.

\begin{tabular}{|c|c|c|c|c|}
\hline \multirow{2}{*}{$\begin{array}{l}\text { Postoperative in-hospital } \\
\text { mortality }\end{array}$} & \multirow{2}{*}{$\begin{array}{l}\text { Patient no. } \\
\quad(n=84)\end{array}$} & \multicolumn{2}{|c|}{ Serum ACE2 levels (ng/ml) } & \multirow[b]{2}{*}{ P-value } \\
\hline & & $\leq 1.34(\mathrm{n}=42)$ & $>1.34(\mathrm{n}=42)$ & \\
\hline Total mortality & $33(39.3)$ & $25(59.5)$ & $8(19.0)$ & $<0.001$ \\
\hline \multicolumn{5}{|l|}{ Cause of mortality } \\
\hline Cardiac AEs & $11(13.1)$ & $9(21.4)$ & $2(4.8)$ & $0.048^{\mathrm{a}}$ \\
\hline Pulmonary AEs & $10(11.9)$ & $9(21.4)$ & $1(2.4)$ & $0.015^{\mathrm{a}}$ \\
\hline Renal failure & $6(8.3)$ & $6(14.3)$ & $0(0.0)$ & $0.026^{\mathrm{a}}$ \\
\hline Other & $6(8.3)$ & $1(2.4)$ & $5(11.8)$ & 0.202 \\
\hline
\end{tabular}

${ }^{\mathrm{a}} \mathrm{P}<0.05$. Data are expressed as the $\mathrm{n}(\%)$. The percentage indicates the individual $\%$ of the total subject number in each column. Other mortality causes included infection and compartment syndrome. ACE2, angiotensin-converting enzyme 2; AE, adverse events.

in Table $\mathrm{V}$, the risk of postoperative in-hospital mortality was markedly higher in the two lowest serum ACE2 level quartiles of $\leq 1.01$ and $1.02-1.34 \mathrm{ng} / \mathrm{ml}$ (HR, 6.1 and 3.4, respectively) when compared with the highest quartile level of $\geq 1.80 \mathrm{ng} / \mathrm{ml}$ $(\mathrm{P}<0.001)$. This finding confirmed that a low serum ACE2 level may be an independent risk factor for increased in-hospital mortality following OSR for ruptured AAA.

Next, the lowest two ACE2 level quartiles were combined into one category $(\leq 1.34 \mathrm{ng} / \mathrm{ml} ; \mathrm{n}=42)$ in order to compare these with the combined highest two ACE2 level quartiles ( $>1.34 \mathrm{ng} / \mathrm{ml} ; \mathrm{n}=42)$. As shown in Table VI, 25 out of $42(59.5 \%)$ patients with a serum ACE2 level $\leq 1.34 \mathrm{ng} / \mathrm{ml}$ succumbed during postoperative hospitalization; by contrast, only 8 in 42 (19.0\%) patients with a serum ACE2 level $>1.34 \mathrm{ng} / \mathrm{ml}$ succumbed during postoperative hospitalization. In addition, stratified analyses of the causes of postoperative in-hospital mortality showed that mortality in patients with a serum ACE2 level $\leq 1.34 \mathrm{ng} / \mathrm{ml}$ was primarily a result of cardiopulmonary adverse events and renal failure. An increased number of patients in the $\leq 1.34 \mathrm{ng} / \mathrm{ml}$ group succumbed due to these causes, when compared with those in the $>1.34 \mathrm{ng} / \mathrm{ml}$ group (Table VI).

\section{Discussion}

OSR is a conventional surgical method performed to repair a ruptured AAA, however it is associated with a high postoperative mortality rate (4). Patients undergoing OSR may succumb within a few days of ICU hospitalization (5). Thus, identifying the risk/predictive factors or biomarkers of postoperative mortality in these patients may lead to adjustment of treatment and improved outcomes. The present study provided evidence supporting that the preoperative serum ACE2 level is an independent risk factor for the in-hospital mortality following OSR for ruptured AAA.

Previous studies have shown significant variations in the serum ACE2 levels prior to and following major cardiopulmonary surgeries (12-14). Wang et al reported that the serum ACE2 level in patients who underwent CABG with cardiopulmonary bypass was increased over the baseline value at $1 \mathrm{~h}$ post-CABG, with the value peaking at 6 days after surgery and returning to the baseline level 12 days after surgery (8). Furthermore, Li et al showed that the serum ACE2 level in NSCLC patients peaked $24 \mathrm{~h}$ after major pulmonary resection and returned to the baseline level 3 days after the surgery (9). In the present study, the serum ACE2 levels remained roughly the same prior to and following OSR for ruptured AAA. The observed discrepancy between the present and previous studies may be due to the different tissues, disease types and surgical techniques involved in these studies. Notably, although there were no significant differences in the general and disease characteristics at baseline, the non-survivors showed consistently significantly lower serum ACE2 levels when compared 
with those in survivors, both before and after the surgery, suggesting that the serum ACE2 level may be associated with in-hospital mortality subsequent to OSR for ruptured AAA.

The current study analyzed the serum ACE2 level on preoperative day 1. Serum ACE2 levels are suggested as a potential biomarker based on the observation that non-survivors consistently showed significantly lower serum ACE2 levels when compared with survivors, both prior to and following the surgery. In addition, there was no significant difference in the mean serum ACE2 levels prior to and following the surgery in non-survivors or survivors. Therefore, preoperative prediction of the mortality risk, based on the serum ACE2 levels, could provide a greater time frame for adjustment of the treatment. In the present study, preoperative day 1 was the last tested time point prior to surgery, on which biochemical assay results would most closely reflect the patient's condition.

A study by Bhak et al (2) demonstrated that the current smoking status and diastolic blood pressure of patients were positively associated with the AAA expansion rate, while diabetes mellitus was negatively associated with the expansion rate. In addition, Steyerberg et al (15) reported in 1995 that age, gender and the presence of cardiac, renal and pulmonary comorbidities were risk factors for perioperative mortality of elective AAA surgery. In order to adjust for the aforementioned potential confounders on the association between the serum ACE2 level and the in-hospital mortality following OSR for ruptured AAA, the present study included age, gender, current smoking status, diabetes mellitus, cardiovascular comorbidity, pulmonary comorbidity and kidney disease, as well as any variables with $\mathrm{P}<0.50$, in the comparisons between non-survivors and survivors as independent variables in multivariate logistic regression analysis. Subsequent to adjusting for the potential confounders, the serum ACE2 level on preoperative day 1 was found to present a significant negative association with the postoperative in-hospital mortality; this suggested that a low serum ACE2 level on preoperative day 1 was an independent risk factor for increased in-hospital mortality following OSR for ruptured AAA. This finding was confirmed by multivariate HR analysis, which showed that, subsequent to adjusting for the potential confounders, the risk of postoperative in-hospital mortality remained significantly higher in the two lowest serum ACE2 level quartiles when compared with that in the highest quartile on preoperative day 1. Therefore, the serum ACE2 level on preoperative day 1 may be a potential novel biomarker or prognostic factor for in-hospital mortality following OSR for ruptured AAA. In view of various commercially available ELISA kits that can readily quantify serum ACE2 levels within $5 \mathrm{~h}$, the serum ACE2 level may be the first potential biomarker for the in-hospital mortality following OSR for ruptured AAA. This finding may have significant clinical application potential, given that a reference value range for the serum ACE2 level on preoperative day 1 is determined in future studies. The observations of the current study will be explored further in future larger patient population studies.

ACE2, which is expressed and active in the majority of tissues, is an essential endogenous regulator of the RAS that serves a crucial role in cardiovascular regulation (6). Previous studies have reported increased morbidities and risk of postoperative myocardial infarction following major cardiopulmonary surgery $(8,9)$. In agreement with previous studies demonstrating the cardiopulmonary protective effects of high levels of serum ACE2 $(8,9)$, the present stratified analysis of postoperative in-hospital mortality causes revealed that patients with lower serum ACE2 levels experienced a significantly higher number of postoperative cardiopulmonary adverse effects and renal failure, which resulted in postoperative mortality. This suggests that increasing ACE2 levels may reduce in-hospital mortality following OSR for ruptured AAA by reducing postoperative cardiopulmonary and renal adverse events. However, the underlying mechanisms of the action of ACE2 remain unclear. In the current study, this action is not likely to be associated with the regulation of blood pressure, since the serum ACE2 level was not correlated with blood pressure, as indicated in correlation and multivariate analyses. Furthermore, this action is not associated with potential regulation of the AAA disease characteristics by serum ACE2, since adjustment for the disease characteristics did not alter the significant association between the serum ACE2 level and postoperative in-hospital mortality. As mentioned earlier, the current study observed decreased serum ACE2 levels in non-survivors, whereas there was no significant difference between the preoperative and postoperative mean levels in non-survivors or survivors. According to these observations, the serum ACE2 is likely to represent a stress hormone, the level of which may determine the efficiency of stress response to major cardiovascular or cardiopulmonary surgery in patients. This concept will be elaborated further in our future studies.

The present study used a relatively large sample size of patients undergoing OSR for ruptured AAA $(n=84)$, which provided adequate statistical accuracy in the multivariate analyses that adjusted for multiple relevant factors. However, the current study had certain limitations. Only the association of serum ACE2 levels with postoperative in-hospital mortality was assessed, thus the long-term association remains unclear. In addition, the current findings may have clinical application potential, but a reference value range for the serum ACE2 level within $24 \mathrm{~h}$ before OSR for AAA has yet to be defined. Using this range, surgeons may potentially identify patients with higher risk of postoperative mortality and thereby adjust clinical management accordingly for better outcomes. This will require a prospective cohort study with a larger patient population for further validation.

In conclusion, the present study provided the first evidence supporting that the serum ACE2 level is an independent risk factor for in-hospital mortality following OSR for ruptured AAA. In particular, low serum ACE2 levels on preoperative day 1 were associated with increased postoperative in-hospital mortality. Therefore, the serum ACE2 level on preoperative day 1 may be the first potential biomarker or prognostic factor for in-hospital mortality subsequent to OSR for ruptured AAA.

\section{References}

1. BurilloE,LindholtJS,Molina-SánchezP,JorgeI,Martinez-PinnaR, Blanco-Colio LM, Tarin C, Torres-Fonseca MM, Esteban M, Laustsen J, et al: ApoA-I/HDL-C levels are inversely associated with abdominal aortic aneurysm progression. Thromb Haemost 113: 1335-1346, 2015. 
2. Bhak RH, Wininger M, Johnson GR, Lederle FA, Messina LM Ballard DJ and Wilson SE: Factors associated with small abdominal aortic aneurysm expansion rate. JAMA Surg 150: 44-50, 2015.

3. Al-Omran M, Verma S, Lindsay TF, Weisel RD and Sternbach Y: Clinical decision making for endovascular repair of abdominal aortic aneurysm. Circulation 110: e517-e523, 2004.

4. Raats JW, Flu HC, Ho GH, Veen EJ, Vos LD, Steyerberg EW and van der Laan L: Long-term outcome of ruptured abdominal aortic aneurysm: Impact of treatment and age. Clin Interv Aging 9: 1721-1732, 2014.

5. Krenzien F, Matia I, Wiltberger G, Hau HM, Schmelzle M Jonas S, Kaisers UX and Fellmer PT: Early prediction of survival after open surgical repair of ruptured abdominal aortic aneurysms. BMC Surg 14: 92, 2014

6. Domenighetti AA, Wang Q, Egger M, Richards SM, Pedrazzini T and Delbridge LM: Angiotensin II-mediated phenotypic cardiomyocyte remodeling leads to age-dependent cardiac dysfunction and failure. Hypertension 46: 426-432, 2005.

7. Raizada MK and Ferreira AJ: ACE2: A new target for cardiovascular disease therapeutics. J Cardiovasc Pharmacol 50: 112-119, 2007.

8. Wang M, Zhang W, Zhou Y and Zhou X: Association between serum angiotensin-converting enzyme 2 levels and postoperative myocardial infarction following coronary artery bypass grafting. Exp Ther Med 7: 1721-1727, 2014.
9. Li X, Zhou $\mathrm{C}$ and $\mathrm{Hu} \mathrm{W}$ : Association between serum angiotensin-converting enzyme 2 level with postoperative morbidity and mortalityafter major pulmonary resection in non-small cell lung cancer patients. Heart Lung Circ 23: 661-666, 2014.

10. Sakalihasan N, Limet R and Defawe OD: Abdominal aortic aneurysm. Lancet 365: 1577-1589, 2005.

11. Yan Y: Logistic regression analysis. In: Medical statistics. Sun ZQ and Xu YY (eds). 2nd edition. People's Medical Publishing House, Beijing, pp292, 2010 (In Chinese).

12. Dahlin LG, Olin C and Svedjeholm R: Perioperative Myocardial Infarction in Cardiac Surgery: Risk Factors and Consequences: A Case Control Study. Scand Cardiovasc J 34: 522-527, 2000.

13. Andesberg G, Beattie WS, Mosseri M, Jaffe AS and Alpert JS: Perioperative Myocardial Infarction. Circulation 119: 2936-2944, 2009.

14. Lim CC,Cuculi F, van Gaal WJ, Testa L, Arnold JR, Karamitsos T, Francis JM, Digby JE, Antoniades C, Kharbanda RK, et al: Early diagnosis of perioperative myocardial infarction after coronary bypass grafting: A study using biomarkers and cardiac magnetic resonance imaging. Ann Thorac Surg 92: 2046-2053, 2011.

15. Steyerberg EW, Kievit J, de Mol Van Otterloo JC, van Bockel JH, Eijkemans MJ and Habbema JD: Perioperative mortality of elective abdominal aortic aneurysm surgery. A clinical prediction rule based on literature and individual patient data. Arch Intern Med 155: 1998-2004, 1995 